\title{
Vector model of the timing diagram of automatic machine
}

\author{
A. Jomartov \\ Institute Mechanics and Mechanical Engineering, Almaty, Kazakhstan \\ Correspondence to: A. Jomartov (legsert@mail.ru)
}

Received: 25 December 2012 - Revised: 11 October 2013 - Accepted: 28 November 2013 - Published: 11 December 2013

\begin{abstract}
In this paper a vector model of timing diagram of automatic machine is developed, which allows us to solve a variety dynamic tasks by changing the parameters of timing diagram of its mechanisms. The connection between the parameters of the timing diagram of automatic machine and equations of motion mechanisms through functions of position and transfer functions of mechanisms is established. The vector model of timing diagram can be used to optimize the timing diagrams of looms and polygraphic machines.
\end{abstract}

\section{Introduction}

Modeling of the timing diagram is one of the main parts for design of automatic machines. A detailed analysis of the works on the theory of the timing diagram performed prior to 1965 is given in Petrokas (1970). Timing diagram is a sequence of machine operations performed by mechanisms depending on the angular displacement of the main shaft (Browne, 1965; Youssef and El-Hofy, 2008; Homer, 1998; Sandler, 1999; Natale C, 2003; Singh and Bhattacharya, 2006; Levner and Kats, 2007; Niir Board, 2009; Norton, 2009; Topalbekiroglu and Celik, 2009). Timing diagram allows determining of the position of each of the executive body at any position of the main shaft (see Fig. 1).

Timing diagram automatic machine is modeled by a directed graph (see Fig. 2) (Novgorodtsev, 1982). The disadvantages of this model are the lack of consideration for connections executive bodies displacements mechanisms and accounting precision of manufacturing.

Analysis of the methods of synthesis and analysis of timing diagram automatic machine showed the need for further development of optimization methods of timing diagram, taking into account the accuracy of manufacture and the dynamics of automatic machine.

\section{Vector model of the timing diagram of automatic machine}

The timing diagram of automatic machines can be represented as the vector polygons (Jomartov, 2010, 2011) (see
Fig. 3). Let us replace the segments linear timing diagram by the vectors $\boldsymbol{\ell}_{i j}$. The vectors $\boldsymbol{\ell}_{i j}$ is directed sequentially from one position to another position of mechanism, where $i$ is the number of mechanisms, $j$ is the number of position of $i$ mechanism, $m_{j}$ is the number of positions of $i$-mechanism, $n$ is the number of mechanisms.

The projection of vectors $\boldsymbol{\ell}_{i j}$ on the $x$ axis is $\alpha_{i j}$-phase angles of actuation of mechanisms. The projection $\boldsymbol{\ell}_{i j}$ on the $y$ axis is the displacement $\delta_{i j}$ of $j$-position of $i$-mechanism.

$\delta_{i j}=\frac{S_{i j}}{S_{\max }}, \quad S_{\max }=\max S_{i j}, \quad i=1, \ldots, n ; j=1, \ldots, m_{i}$,

where $S_{i j}$ is the displacement of $j$-position of $i$-mechanism.

To explain the parameters $\alpha_{i j}$ and $S_{i j}$ in Fig. 4 shows a diagram of the displacement of mechanism, in the figure denote: $\alpha_{\mathrm{o}}$ - phase angles of actuation of mechanism in the position of open, $\alpha_{\mathrm{d}}$ is the phase angles of actuation of mechanism in the position of dwell, $\alpha_{\mathrm{c}}$ is the phase angles of actuation of mechanism in the position of close, $S_{\mathrm{o}}$ is the displacement of mechanism in the position of open, $S_{\mathrm{c}}$ is the displacement of mechanism in the position of close.

Let us introduce the vector $\boldsymbol{P}$ connecting the point of beginning and end of the cycle. The projection of the vector $\boldsymbol{P}$ on the $x$ axis is $2 \pi$ on the $y$ axis is zero. The interaction of mechanisms with each other will reflect in the form of the vectors of connection $\boldsymbol{c}_{i k}$, where $k=1, \ldots, r_{i}, r_{i}$ is the number of vectors of connection of $i$-mechanism. The projection of the vectors of connection to the $x$ axis is the delay of actuation mechanism, and the projection on the $y$ axis is the difference between the displacements of mechanisms. 


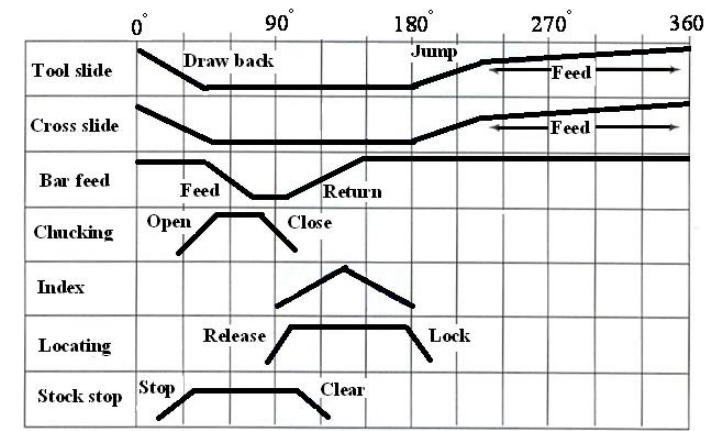

Figure 1. Linear timing diagram of working and auxiliary cams of a four-spindle bar automatic.

Let us impose the timing diagram of mechanisms at each other using zero vectors (see Fig. 3) connecting the boundary points of timing diagram mechanisms in the $y$ axis.

Let us compose the system of vector equations describing the works of mechanisms automatic machine (see Fig. 3).

$$
\left.\begin{array}{l}
\sum_{j=1}^{m_{i}} \boldsymbol{\ell}_{i j}=\boldsymbol{P}, i=1, \ldots, n, \\
\boldsymbol{c}_{i k}=\sum_{i=1}^{n} \sum_{j=1}^{m_{i}} b_{i j} \cdot \boldsymbol{\ell}_{i j}
\end{array}\right\},
$$

where $b_{i j} \in\{0, \pm 1\}$.

Let us projected the vector Eq. (1) on the axis $x$ and $y$.

$$
\left.\begin{array}{l}
\sum_{j=1}^{m_{i}} \alpha_{i j}=2 \pi, \sum_{j=1}^{m_{i}} \delta_{i j}=0, \\
c_{i k}^{x}=\sum_{i=1}^{n} \sum_{j=1}^{m_{i}} b_{i j} \alpha_{i j}, c_{i k}^{y}=\sum_{i=1}^{n} \sum_{j=1}^{m_{i}} b_{i j} \delta_{i j}
\end{array}\right\}
$$

On the phase angles of actuation of mechanisms $\alpha_{i j}$, and displacements of mechanisms $\delta_{i j}$ impose constraints

$\alpha_{i j} \geq \alpha_{i j}^{\mathrm{m}}, \delta_{i j}^{\ell} \geq \delta_{i j} \geq \delta_{i j}^{\mathcal{H}}$,

where $\alpha_{i j}^{\mathrm{m}}$ is the minimum allowable phase angles of actuation of mechanisms, $\delta_{i j}^{\ell}$, $\delta_{i j}^{\mathcal{H}}$ is the upper and lower limits assigned by the designer.

On the projection vectors of connection impose constraints

$\boldsymbol{c}_{i k}^{x \ell} \geq \boldsymbol{c}_{i k}^{x} \geq \boldsymbol{c}_{i k}^{x \ell}, \boldsymbol{c}_{i k}^{y \ell} \geq \boldsymbol{c}_{i k}^{y} \geq \boldsymbol{c}_{i k}^{y \mathcal{H}}$

where $\boldsymbol{c}_{i k}^{\chi \mathcal{H}}=\boldsymbol{e}_{i k}^{x}+\Delta \boldsymbol{c}_{i k}^{x}, \boldsymbol{c}_{i k}^{y \mathcal{H}}=\boldsymbol{e}_{i k}^{y}+\Delta \boldsymbol{c}_{i k}^{y}$ where $\boldsymbol{e}_{i k}^{x}, \boldsymbol{e}_{i k}^{y}$ are the minimum permissible projection vectors of connection, $\Delta \boldsymbol{c}_{i k}^{x}$, $\Delta \boldsymbol{c}_{i k}^{y}$ are the errors of the projections of vectors of connection, $\boldsymbol{c}_{i k}^{x \ell}, \boldsymbol{c}_{i k}^{y \ell}$ are the upper limits imposed by the designer.

Equation (2) and constraints (Eqs. 3 and 4) describe the collaboration works of mechanisms (timing diagram) of automatic machine.

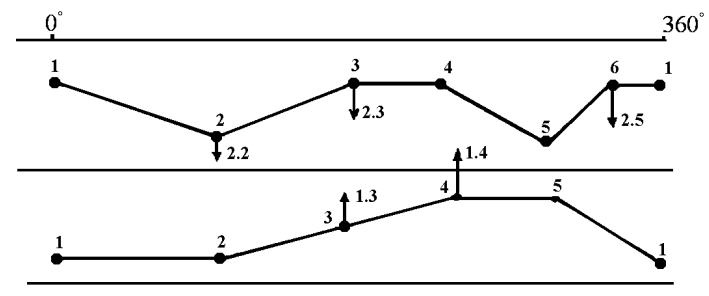

Figure 2. Presentation of timing diagram automatic machine as a directed graph.

\section{A mathematical model of automatic machine based on the timing diagram of mechanisms}

Lets define the connection between the differential equations of motion the automatic machine and the equations describing its timing diagram. In Fig. 5 shows the dynamic model of the machine, where $c_{i}$ is the elasticity coefficients, $\beta_{i}$ is the coefficients of resistance, $J_{\mathrm{i}}, I_{\mathrm{i}}$ are the moments of inertia, $M_{\partial B}$ is the motor torque, $M_{i}$ is moment of resistance, $\Pi_{i}$, $i=1, \ldots, n$ is the function of position of mechanisms.

To compile the equations of motion mechanisms automatic machine (see Fig. 5), let us use Lagrange equations II (Wolfson, 1976):

$$
\left.\begin{array}{l}
\frac{\mathrm{d}}{\mathrm{d} t}\left(\frac{\partial T}{\partial \dot{\varphi}_{j}}\right)-\frac{\partial T}{\partial \varphi_{j}}+\frac{\partial V}{\partial \varphi_{j}}=Q_{j}+\sum_{i=1}^{m} \lambda_{i} h_{i j} \\
\sum_{j=1}^{m+n} h_{i j} \dot{\varphi}_{j}+h_{i}=0,
\end{array}\right\}
$$

where $\varphi_{1}, \varphi_{2}, \ldots, \varphi_{n}$ are the generalized coordinates, $\lambda_{i}$ is Lagrange multipliers, $h_{i j}, h_{i}$ are some functions, $T$ is the kinetic energy of a holonomic system, $V$ is the potential energy of the system, $Q_{j}$ is the generalized force.

To establish the connection between the equations of timing diagram (2-4) and the dynamic Eq. (5), let us write the functions of position, the transfer functions of mechanisms of automatic machine in the following form:

$$
\left.\begin{array}{l}
\Pi_{i}=\Pi_{i 1} \cdot\left[1-L\left(\phi_{i}-\alpha_{i 1}\right)\right]+\sum_{j=2}^{m} \Pi_{i j}\left[1-L\left(\phi_{i}-\sum_{r=1}^{j} \alpha_{i r}\right)\right] \cdot L\left(\phi_{i}-\sum_{r=1}^{j-1} \alpha_{i r}\right) \\
\Pi_{i}^{\prime}=\Pi_{i 1}^{\prime}\left[1-L\left(\phi_{i}-\alpha_{i 1}\right)\right]+\sum_{j=2}^{m} \Pi_{i j}^{\prime}\left[1-L\left(\phi_{i}-\sum_{r=1}^{j} \alpha_{i r}\right)\right] \cdot L\left(\phi_{i}-\sum_{r=1}^{j=1} \alpha_{i r}\right) \\
\Pi_{i}^{\prime \prime}=\Pi_{i 1}^{\prime \prime}\left[1-L\left(\phi_{i}-\alpha_{i 1}\right)\right]+\sum_{j=2}^{m} \Pi_{i j}^{\prime \prime}\left[1-L\left(\phi_{i}-\sum_{r=1}^{j} \alpha_{i r}\right)\right] \cdot L\left(\phi_{i}-\sum_{r=1}^{j-1} \alpha_{i r}\right)
\end{array}\right\}
$$

where $i=1, \ldots, n, L(x)$ is a step function of the form

$L(x)=\left\{\begin{array}{c}0, x<0 \\ 1, x \geq 0\end{array}\right.$.

$\Pi_{i j}, \Pi_{i j}^{\prime}, \Pi_{i j}^{\prime \prime}$ are the functions of position, the first transfer function, the second transfer function on parts of phase angles of actuation $\alpha_{i j}$ of mechanisms.

Equation (6) establish a connection between the Eq. (5) describe the dynamics of the automatic machine and the Eqs. (2)-(4) timing diagram of the machine-automaton. This 


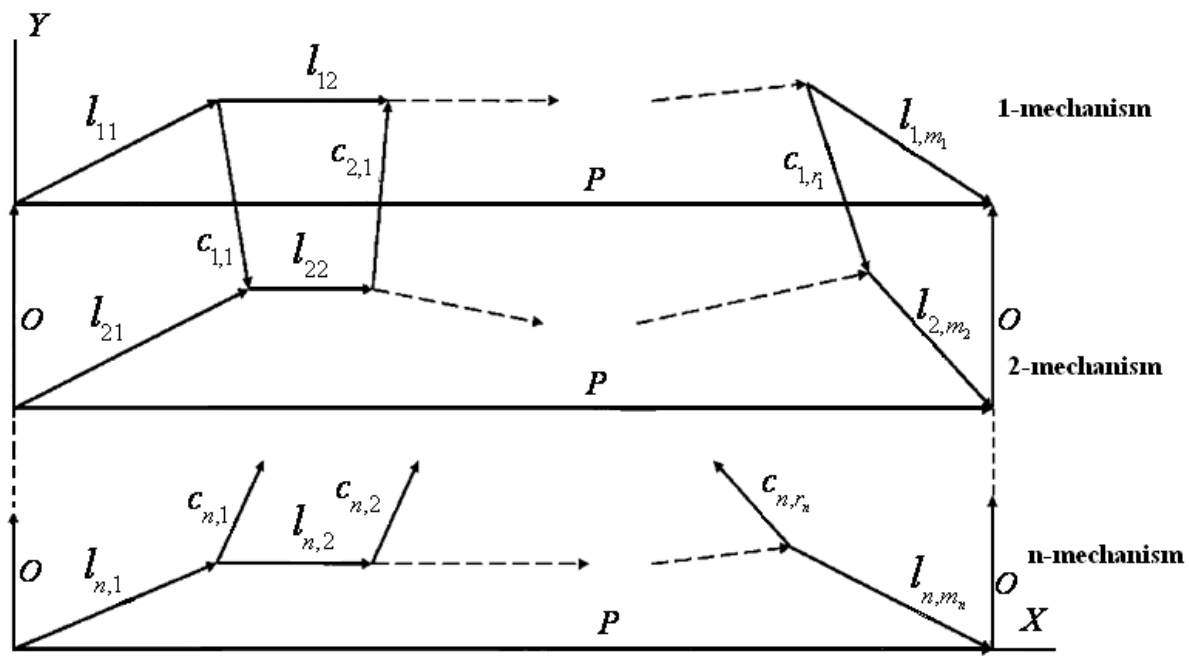

Figure 3. Vector model of the timing diagram of automatic machine.

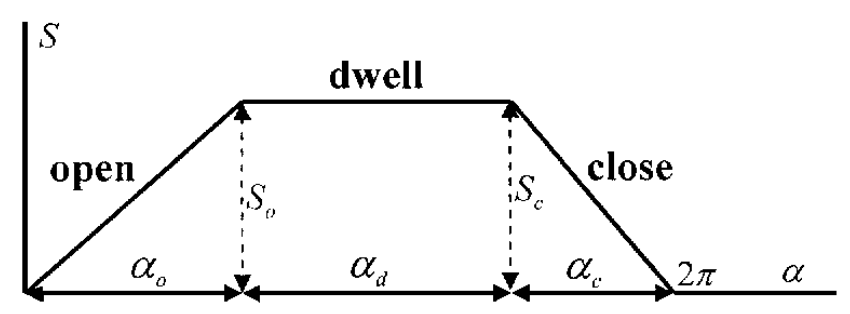

Figure 4. Diagram of the displacement of mechanism.

method allows to solve various optimization tasks, where the variable parameters are the phase angles $\alpha_{i j}$ and of the displacements $\delta_{i j}$ of timing diagram automatic machine.

\section{An example}

Let us show in more detail the connection between timing diagram of automatic machine and dynamics of mechanisms on the example of automatic machine with two cam mechanisms. The dynamic model is shown in Fig. 6, where $\varphi_{0}, \varphi_{1}$, $\varphi_{2}$ are generalized coordinates, $M_{D}$ is the motor torque, $M_{1}$, $M_{2}$ are the moments of resistance, $I_{1}, J_{0}, J_{1}, J_{2}$ are the moments of inertia of mechanisms, $c_{0}, c_{1}$ are the coefficients of elasticity of shafts, $\Pi_{i}\left(\phi_{i}\right)$ is the function of position of cam mechanisms, $\Pi_{i}^{\prime}\left(\phi_{i}\right)$ is the first transfer functions of the cams, $\Pi_{i}^{\prime \prime}\left(\phi_{i}\right)$ is the second transfer functions of the cams.

This dynamic model is described by the following equations

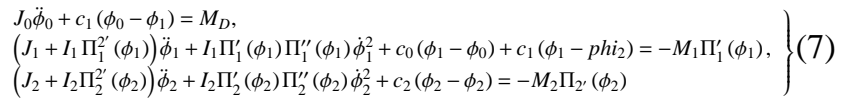

where
$\Pi_{i}^{\prime}\left(\phi_{i}\right)=\frac{d \Pi_{i}\left(\phi_{i}\right)}{d \phi_{i}} ; \Pi_{i}^{\prime \prime}\left(\phi_{i}\right)=\frac{d^{2} \Pi_{i}\left(\phi_{i}\right)}{d \phi_{i}^{2}} ; i=1,2$.

Figure 7 shows vector timing diagram of automatic machine, which is described by the following equations:

$$
\left.\begin{array}{l}
\boldsymbol{l}_{11}+\boldsymbol{l}_{12}=\boldsymbol{P} \\
\boldsymbol{l}_{21}+\boldsymbol{l}_{22}=\boldsymbol{P} \\
\boldsymbol{c}_{21}=\boldsymbol{l}_{21}-\boldsymbol{l}_{11}
\end{array}\right\} \text {. }
$$

Let us projected Eq. (8) on the $x, y$ respectively

$$
\begin{aligned}
& \left.\begin{array}{l}
\alpha_{11}+\alpha_{12}=2 \pi \\
\alpha_{21}+\alpha_{22}=2 \pi \\
c_{11}^{x}=\alpha_{21}-\alpha_{11}
\end{array}\right\} \\
& \delta_{11}-\delta_{12}=0 \\
& \left.\begin{array}{l}
\delta_{21}-\delta_{22}=0 \\
c_{11}^{y}=\delta_{21}-\delta_{11}
\end{array}\right\} .
\end{aligned}
$$

Impose the constraints on the phase angles, displacements of mechanisms, and projections of vectors of connection

$$
\left.\begin{array}{l}
\alpha_{i j} \geq \alpha_{i j}^{\min } \\
\delta_{i j}^{\max } \geq \delta_{i j} \geq \delta_{i j}^{\min } \\
c_{11}^{x \max } \geq c_{11}^{x} \geq c_{11}^{x \min } \\
c_{11}^{y \max } \geq c_{11}^{y} \geq c_{11}^{y \min }
\end{array}\right\} .
$$

The expressions (Eqs. 9-11) allow you to vary the phase angles and displacements of mechanisms of automatic machine, without disrupting their normal work.

To establish the connection between the equations describing of joint work of the mechanisms of automatic machine (Eqs. 9-11) and the dynamic Eq. (7), let us write the functions of position and the transfer functions of mechanisms of automatic machine (see Fig. 8) as follows: 


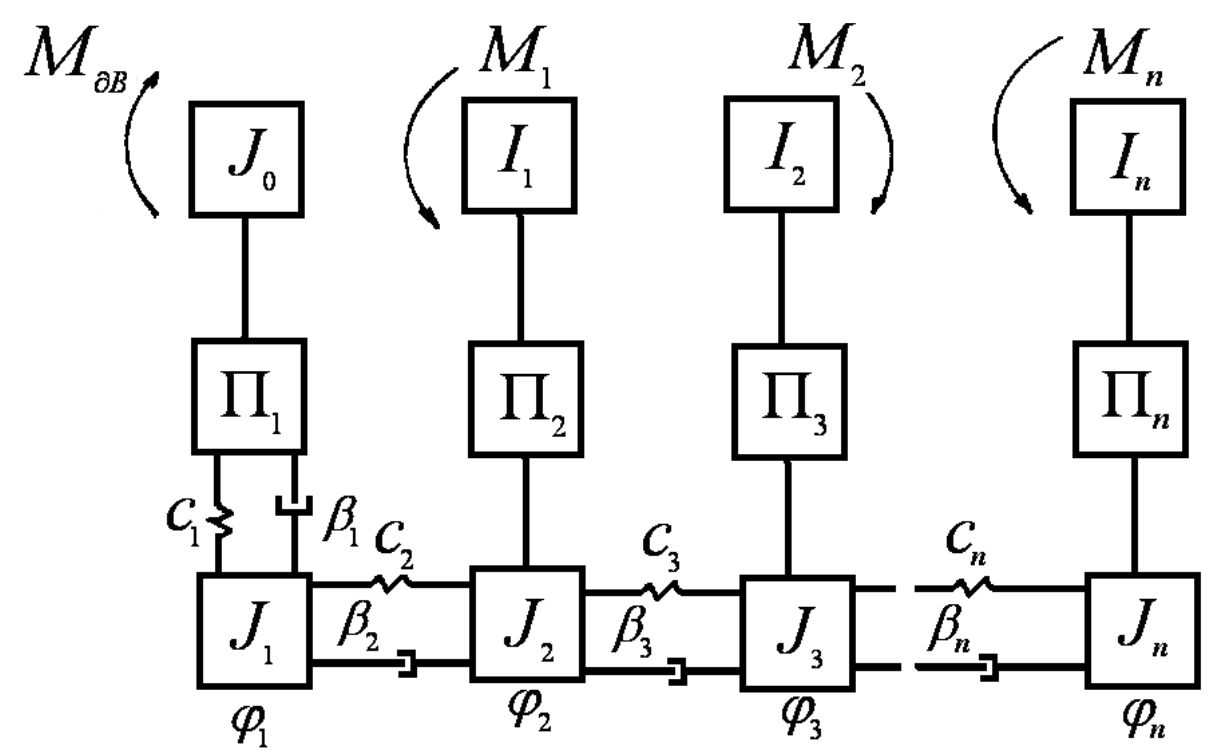

Figure 5. A dynamic model of automatic machine.

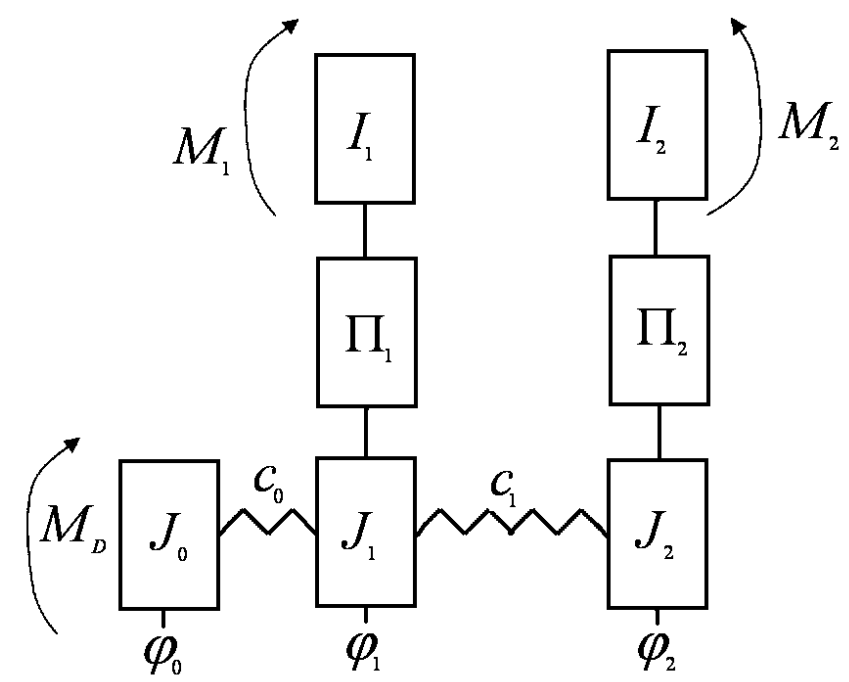

Figure 6. Dynamic model of automatic machine with two cams mechanisms.

$$
\left.\begin{array}{l}
\Pi_{i}=\Pi_{i 1} \cdot\left[1-L\left(\phi_{i}-\alpha_{i 1}\right)\right]+\Pi_{i 2}\left[1-L\left(\phi_{i}-\left(\alpha_{i 1}+\alpha_{i 2}\right)\right)\right] \cdot L\left(\phi_{i}-\alpha_{i 1}\right) \\
\Pi_{i}^{\prime}=\Pi_{i 1}^{\prime 1} \cdot\left[1-L\left(\phi_{i}-\alpha_{i 1}\right)\right]+\Pi_{i 2}^{\prime}\left[1-L\left(\phi_{i}-\left(\alpha_{i 1}+\alpha_{i 2}\right)\right)\right] \cdot L\left(\phi_{i}-\alpha_{i 1}\right) \\
\Pi_{i}^{\prime \prime}=\Pi_{i 1}^{\prime \prime} \cdot\left[1-L\left(\phi_{i}-\alpha_{i 1}\right)\right]+\Pi_{i 2}^{\prime \prime}\left[1-L\left(\phi_{i}-\left(\alpha_{i 1}+\alpha_{i 2}\right)\right)\right] \cdot L\left(\phi_{i}-\alpha_{i 1}\right)
\end{array}\right\}
$$

where

$L(x)=\left\{\begin{array}{c}0, x<0 \\ 1, x \geq 0\end{array}\right.$

Represent the generalized coordinates $\varphi_{1}, \varphi_{2}$ through the dimensionless coefficients $k_{1}, k_{2}$ where $\varphi_{1}=2 \pi k_{1} ; \varphi_{2}=2 \pi k_{2}$; $k_{1}, k_{2} \in[0,1]$

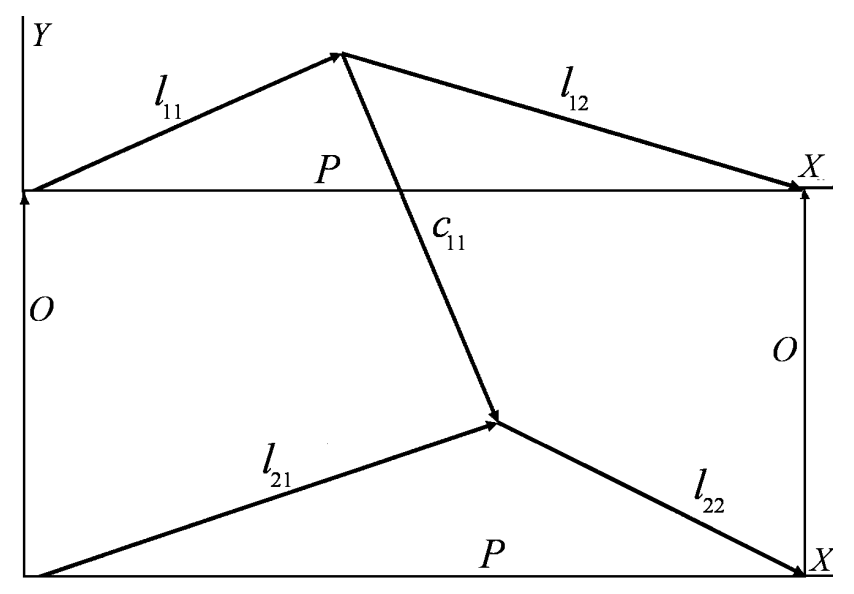

Figure 7. Vector timing diagram of automatic machine with two cams mechanisms.

$$
\left.\begin{array}{c}
\Pi_{i j}=a_{i j}\left(k_{i}\right) \delta_{i j} \\
\Pi_{i j}^{\prime}=b_{i j}\left(k_{i}\right) \frac{\delta_{i j}}{\alpha_{i j}} \\
\Pi_{i j}^{\prime \prime}=d_{i j}\left(k_{i}\right) \frac{\delta_{i j}}{\alpha_{i j}^{2}}
\end{array}\right\} .
$$

$a_{i j}\left(k_{i}\right), b_{i j}\left(k_{i}\right), d_{i j}\left(k_{i}\right) ; i=1,2 ; j=1,2$; are coefficients of the displacement, the velocity, the acceleration of mechanism in $j$-position.

Equations (12) and (13) establish a connection between the phase angles of actuation of mechanisms $\alpha_{i j}$ and magnitude of displacement of mechanisms $\delta_{i j}$ and of their functions of position and transfer functions that are explicitly included in the equations of motion of the automatic machine (Eq. 7). Now, depending on the chosen optimization criterion, by 


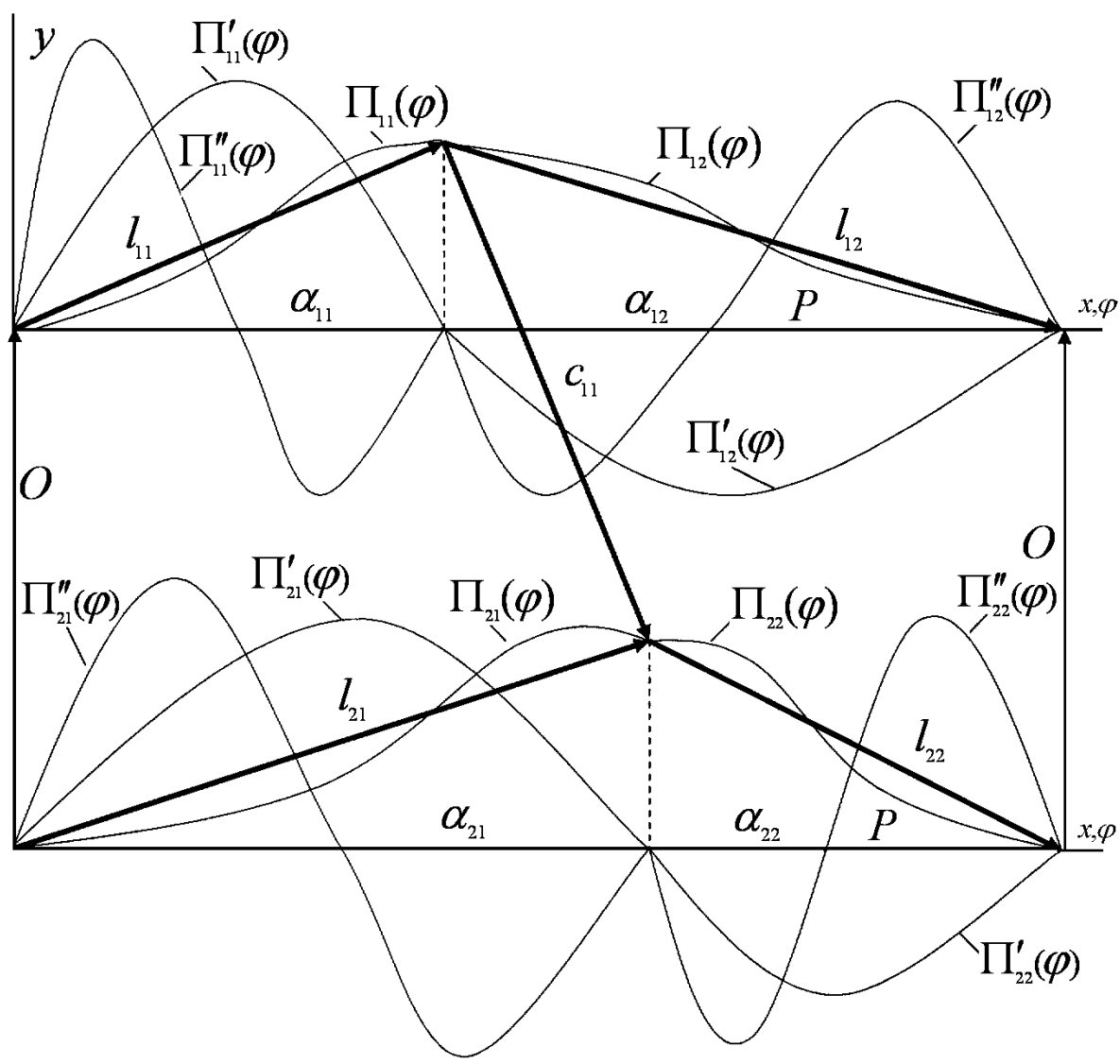

Figure 8. The functions of position and the transfer functions of mechanisms of automatic machine.

varying the parameters of timing diagram $\alpha_{i j}$ and $\delta_{i j}$, can improve the dynamics of the automatic machine. As an optimization criterion can use the following expression:

$$
\max _{\varphi_{i}}\left(\Pi_{i}^{\prime}\left(\varphi_{i}\right) \Pi_{i}^{\prime \prime}\left(\varphi_{i}\right)\right)
$$

\section{Conclusions}

The vector model of timing diagram is developed on the basis of representation timing diagram of the automatic machine as vector polygons, which allows solving various dynamic tasks at the expense of change of parameters of timing diagram.

The mathematical model of the automatic machine with elastic links on the basis of the timing diagram of its mechanisms is received.

The equations of connection between parameters timing diagram of the automatic machine and equations of dynamics through functions of position and transfer functions of mechanisms were received.

The model of timing diagram of the automatic machine is the only method which allows to solve a variety of dynamic tasks, by optimizing its timing diagrams, at this time.
Edited by: A. Barari

Reviewed by: two anonymous referees

\section{References}

Browne, J. W.: The Theory of Machine Tools, Cassell and Co. Ltd., London, p. 374, 1965.

Homer, D. E.: Kinematic Design of machines and mechanisms, McGraw-Hill, New York, p. 661, 1998.

Jomartov, A.: Dynamics of machine-automaton jointly with cyclegram, World Congress on Engineering, Lîndon, UK, 1224-1229, 2010.

Jomartov, A.: Multi-Objective Optimization Of Cyclogram Mechanisms Machine-Automaton, World Congress on Engineering, Lîndon, UK, 2562-2565, 2011.

Levner, E. and Kats, V. D.: Cyclic Scheduling in Robotic Cells:An Extension of Basic Models in Machine Scheduling Theory, Multiprocessor Scheduling: Theory and Applications, Vienna, Austria, p. 436, 2007.

Natale, C.: Interaction control of robot manipulators: six degreesof-freedom tasks, Springer-Verlag, Berlin, p. 108, 2003.

Niir Board: Complete technology book on textile, spinning, weaving, finishing and printing, National Institute of Industrial Re, Delhi, p. 814, 2009. 
Norton, R. L.: Cam Design and Manufacturing Handbook, Industrial Press Inc., p. 591, 2009.

Novgorodtsev, V. A.: Presentation of the machine timing diagram as a graph, Theory of mechanisms and machines, Kharkov, 33, 57-60, 1982.

Petrokas, L. V.: Reviews of timing diagram manufacturing machines and automatic production lines, J. Theory of automatic machines and pneumatic, Moscow, 22-36, 1970.

Sandler, B. Z.: Robotics: designing the mechanisms for automated machinery, Academic Press, San Diego, p. 433, 1999.
Singh, B. and Bhattacharya, S. K.: Control of machines, New Age International, New Delhi, p. 338, 2006.

Topalbekiroglu, M. and Celik, H. I.: Kinematic analysis of beatup mechanism used for handmade carpet looms, Indian J. Fibre Textile Res., 34, 129-136, 2009.

Wolfson, I. I.: Dynamics calculations of cycle mechanisms, Mashinostroenie, Leningrad, p. 328, 1976.

Youssef, H. A. and El-Hofy, H.: Machining technology: machine tools and operations, Taylor \& Francis Group, London, p. 672, 2008. 